\title{
FENOLOGIA REPRODUTIVA E DISPONIBILIDADE DE FRUTOS DE ESPÉCIES ARBÓREAS EM MATA CILIAR NO RIO FORMOSO, MATO GROSSO DO SUL ${ }^{1}$
}

\author{
Paula Reys ${ }^{2,5}$, Mauro Galetti ${ }^{3}$ L. Patrícia C. Morellato $^{2}$ E José Sabino $^{4}$ \\ Biota Neotropica v5 (n2)-http://www.biotaneotropica.org.br/v5n2/pt/abstract?short-communication+bn01205022005 \\ Recebido em 10/12/04. \\ Revisada em 14/06/05. \\ Publicado em 01/07/2005.
2 - Universidade Estadual Paulista, IB, Departamento de Botânica e Grupo de Fenologia e Dispersão de Sementes, Caixa Postal 199, 13506-900 Rio Claro, SP, Brasil.
3- Universidade Estadual Paulista, IB, Departamento de Ecologia e Grupo de Fenologia e Dispersão de Sementes, CEP 13506-900 Rio Claro, SP, Brasil.
4 - UNIDERP, Laboratório de Biodiversidade, Ecologia e Conservação de Ecossistemas Aquáticos CEP 79790-000,
Campo Grande, MS, Brasil.
5 - Autor para correspondência: preys@rc.unesp.br

\begin{abstract}
Phenological studies are important to the understanding of the dynamic of forest ecosystems, especially when they evaluate the availability of fruits to the fauna. The gallery forests occur along water streams and play an important role as natural corridors, allowing the gene flow and connecting populations isolated due the forest fragmentation. The present study aimed to understand how the reproductive phenology and the fruit availability of gallery forest species changes between seasons and over the year. Monthly observations were carried out on 29 plant species, 31\% wind dispersed and $69 \%$ animal dispersed, from the gallery forest of Formoso River, Bonito Municipality, Mato Grosso do Sul, Brazil. Flowering occurred in the transition from dry to wet season, and fruiting mainly during the wet season, but did not present significant correlation to rainfall or temperature, regardless the seasonal pattern. The species Attalea phalerata and Guibourtia hymenifolia accounted for most of the fruit production in the dry season, supplying food resources to frugivores during this period of fruit scarcity.
\end{abstract}

Key words: Anemochory, frugivory, gallery forest, phenology, seed dispersal, zoochory.

\section{Resumo}

Estudos fenológicos são importantes para o entendimento da dinâmica dos ecossistemas florestais, principalmente quando avaliam a disponibilidade de frutos para a fauna. As matas ciliares são formações vegetais que ocorrem ao longo dos cursos d'água e desempenham papel importante na formação dos corredores de fluxo gênico, podendo interligar populações vegetais que foram separadas pelo processo de fragmentação. O presente trabalho procurou entender como varia a fenologia reprodutiva e a disponibilidade de frutos de espécies arbóreas em mata ciliar do rio Formoso, município de Bonito, Mato Grosso do Sul, entre estações e ao longo de um ano. Foram feitas observações mensais em 29 espécies vegetais, 31\% anemocóricas e 68,9\% zoocóricas, das fenofases floração e frutificação, e estimada a produção de frutos por contagem no chão da floresta. A floração ocorreu principalmente na transição entre as estações seca e chuvosa e a frutificação na estação chuvosa e não apresentaram correlações significativas com a pluviosidade ou a temperatura, apesar do padrão sazonal. As espécies Attalea phalerata e Guibourtia hymenifolia destacaram-se pelo fornecimento de frutos para a fauna de frugívoros na estação seca, período de maior escassez de frutos na mata ciliar.

Palavras-chave: Anemocoria, dispersão de sementes, fenologia, frugivoria, mata ciliar, zoocoria.

\footnotetext{
${ }^{1}$ Parte da dissertação de mestrado de P. Reys.
} 


\section{Introdução}

Os estudos fenológicos contribuem para o entendimento da dinâmica dos ecossistemas florestais e são essenciais para o estudo de ecologia e evolução dos trópicos (Croat 1969, Fournier \& Charpantier 1975). Os padrões fenológicos reprodutivos podem ser influenciados por uma série de fatores abióticos como pluviosidade, temperatura e comprimento do dia (Opler et al. 1976, Ashton et al. 1988, van Schaik 1986, Morellato et al. 1989, 2000); modos de dispersão (Charles-Dominique et al. 1981, Wheelwright 1985, Morellato \& Leitão Filho 1992); fatores filogenéticos (Kochmer \& Handel 1986) e atividade de polinizadores e dispersores de sementes (Snow 1965, Rathke \& Lacey 1985).

Alguns trabalhos de fenologia abordam a disponibilidade de frutos para os animais (French 1992, Peres 1994), e em outros, esta avaliação é feita a partir do padrão de frutificação de espécies zoocóricas (Morellato \& Leitão Filho 1992, Griz \& Machado 2001, Batalha \& Martins 2004). Estas espécies, com frutos dispersos por animais, são muito importantes para a manutenção da oferta de recursos para a fauna ao longo do ano (Figliolia \& Kageyama 1995), principalmente em formações vegetais sob clima sazonal, onde ocorrem períodos de escassez de frutos (Galetti \& Pedroni 1994, Develey \& Peres 2000). Por outro lado, os frugívoros são imprescindíveis como agentes efetivos na dispersão das sementes dessas espécies, levando-as a longa distância, e possibilitando a sua regeneração e a colonização de outras áreas (Figliolia \& Kageyama 1995).

As matas ciliares são formações vegetais que ocorrem ao longo dos cursos d’água e desempenham papel importante na formação dos corredores de fluxo gênico, podendo interligar populações vegetais que foram separadas pelo processo de fragmentação (Macedo 1993, Kageyama \& Gandara 2001). Além disso, estas matas contribuem para a estabilização das margens dos rios, o tamponamento e filtragem de nutrientes e/ou agrotóxicos, a interceptação e absorção da radiação solar e para o fornecimento de abrigo e/ou alimento para a fauna aquática e terrestre (Marinho Filho \& Reis 1989, Petts 1990, Sabino \& Castro 1990). Desse modo, recentemente as matas ciliares têm recebido maior atenção, principalmente pelo seu estado crítico de degradação (Oliveira-Filho 1989, Rodrigues 1989, Rodrigues \& Gandolfi 2001). Devido à sua grande importância no que diz respeito à conservação da biodiversidade e à manutenção do equilíbrio dos ecossistemas da biosfera, seu manejo e recuperação foi incluído como uma das prioridades no Programa das Nações Unidas para o Meio Ambiente (PNUMA) (Barbosa 2001).

A maioria dos estudos em matas ciliares brasileiras aborda a florística e estrutura da vegetação (Gibbs \& Leitão Filho 1978, Ratter 1980, Bertoni \& Martins 1987, Catharino 1989, Rodrigues 1989, Oliveira-Filho 1989, Oliveira-Filho et al. 1990, Guarim Neto et al. 1996, Felfili \& Silva Jr. 1992, Felfili et al. 2000, Marimon \& Lima 2001, Marimon et al. 2002). Poucos trabalhos enfocam aspectos de sua ecologia e dinâmica (Oliveira \& Moreira 1992, Felfili 1997), sendo os padrões fenológicos, o espectro de dispersão de sementes das espécies e a avaliação da disponibilidade de frutos, aspectos praticamente desconhecidos ou nunca estudados (Oliveira \& Moreira 1992, Carmo \& Morellato 2001, Funch et al. 2002).

O presente estudo avaliou os padrões fenológicos reprodutivos e a produção de frutos dispersos por animais, em espécies arbóreas da mata ciliar do rio Formoso (MS), verificando sua relação com os fatores climáticos locais e procurando entender a dinâmica destas formações vegetais, respondendo a seguinte questão: existe sazonalidade nos padrões fenológicos reprodutivos, especialmente na oferta de frutos zoocóricos, em mata ciliar no Centro-Oeste do Brasil? Esperamos um padrão sazonal, como observado para outras florestas ciliares no sudeste e nordeste do Brasil, relacionado a sazonalidade climática (Carmo \& Morellato 2001, Funch et al. 2002).

\section{Material e Método}

\section{1 Área de estudo}

O presente estudo foi desenvolvido na fazenda Baía Bonita (56 25' W; $21^{\circ} 09^{\prime} \mathrm{S}$ ), no município de Bonito, localizado na Serra da Bodoquena, sudoeste do estado do Mato Grosso do Sul, região Centro-Oeste do Brasil. Esta fazenda tem 80 ha e possui o atrativo turístico conhecido como “Aquário Natural”, que recebe visitas ordenadas de turistas o ano todo. O trecho de $1.190 \mathrm{~m}$ do rio Formoso que foi estudado atravessa a fazenda, sendo caracterizado por águas claras, leito argilo-arenoso, serapilheira espessa e densa mata ciliar que, em alguns trechos, possui cerca de $500 \mathrm{~m}$ de largura a partir do leito do rio.

\subsection{Clima}

De acordo com sistema de Köeppen (1948) o município de Bonito tem um clima classificado como tropical com inverno seco (Aw), apresentando estação chuvosa no verão e nítida estação seca no inverno, sendo julho o mês mais seco. O diagrama climático para o período de 19 anos na região de Bonito (Figura 1) mostra que a estação seca ocorre de junho a setembro e a estação úmida de outubro a maio. A média anual de pluviosidade e mensal de temperatura foram respectivamente $1366 \mathrm{~mm}$ e $26,1^{\circ} \mathrm{C}$ para o período de 19 anos. No período de estudo (julho de 2000 a junho de 2001) o clima se apresentou mais úmido (precipitação total anual $1587 \mathrm{~mm}$ ), quando comparado à média climática, sendo que os meses mais secos foram de julho a setembro de 2000 e abril a junho de 2001, com seca mais acentuada em abril e maio de 2001, e os meses mais úmidos de outubro de 2000 a março de 2001, com o pico de pluviosidade em outubro. Os dados meteorológicos foram obtidos na estação meteorológica pertencente à Cooperativa Agrícola de Bonito (COOAGRI), localizada a cerca de quatro quilômetros da área de estudo. 

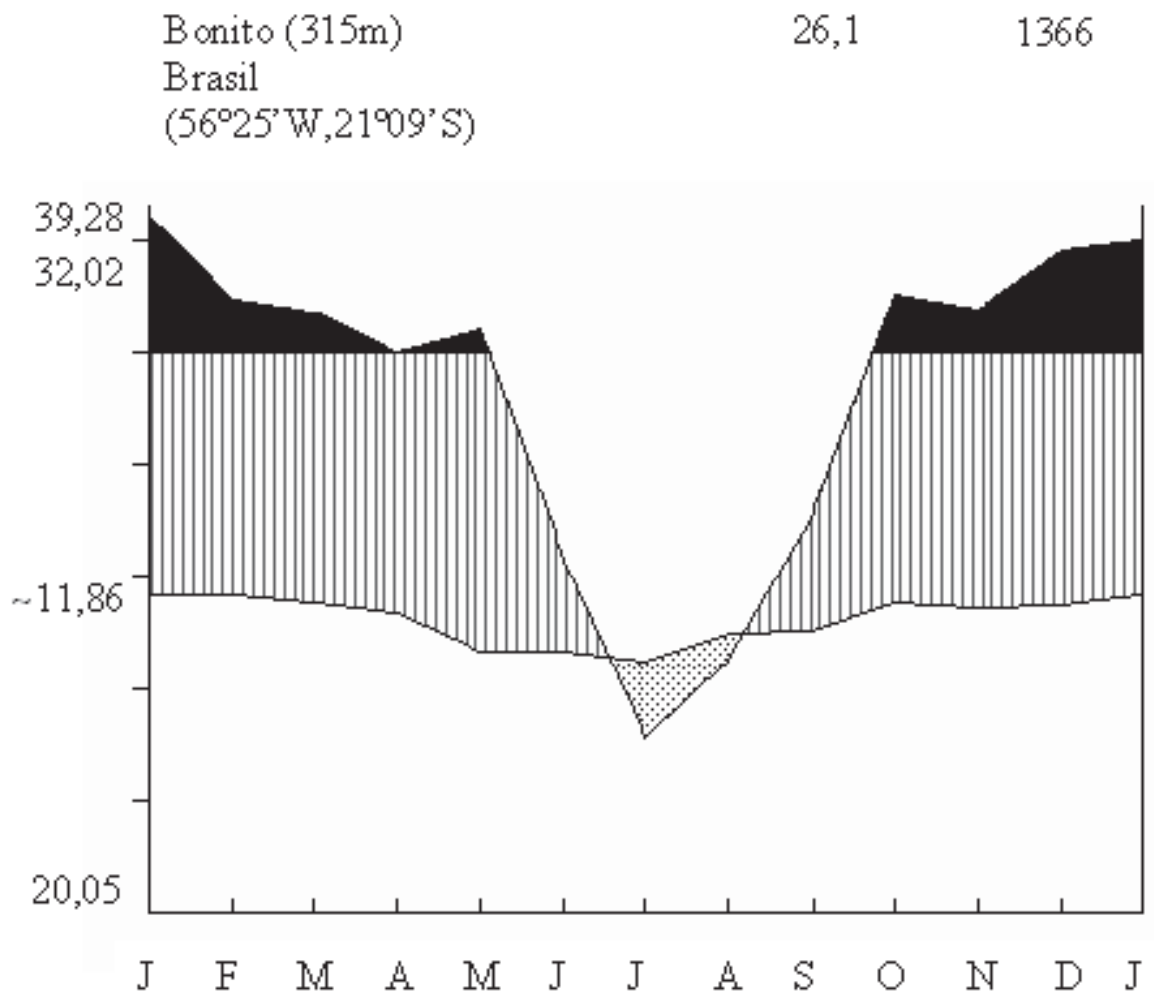

Figura 1 - Diagrama climático de Bonito, Mato Grosso do Sul, para o período de 1982 a 2002. Fonte: Estação Meteorológica da Cooperativa Agrícola de Bonito (COAGRI), localizada em Bonito, Mato Grosso do Sul (56 $25^{\prime} \mathrm{W} 21^{\circ} 09^{\prime} \mathrm{S}, 315 \mathrm{~m}$ de altitude).
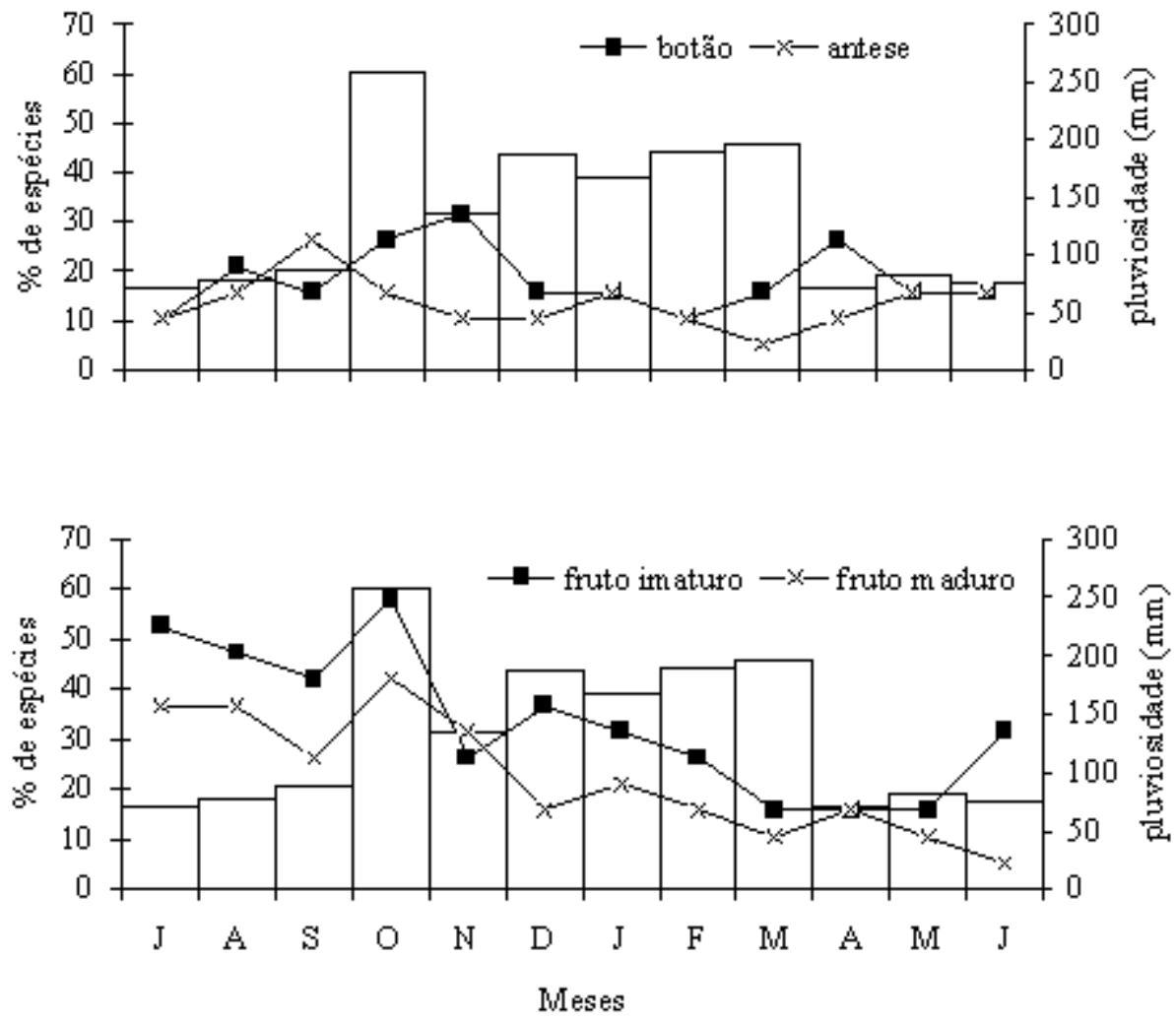

Figura 2 - Porcentagem de espécies em floração (A) e frutificação (B) em mata ciliar do rio Formoso, Mato Grosso do Sul, Brasil. 


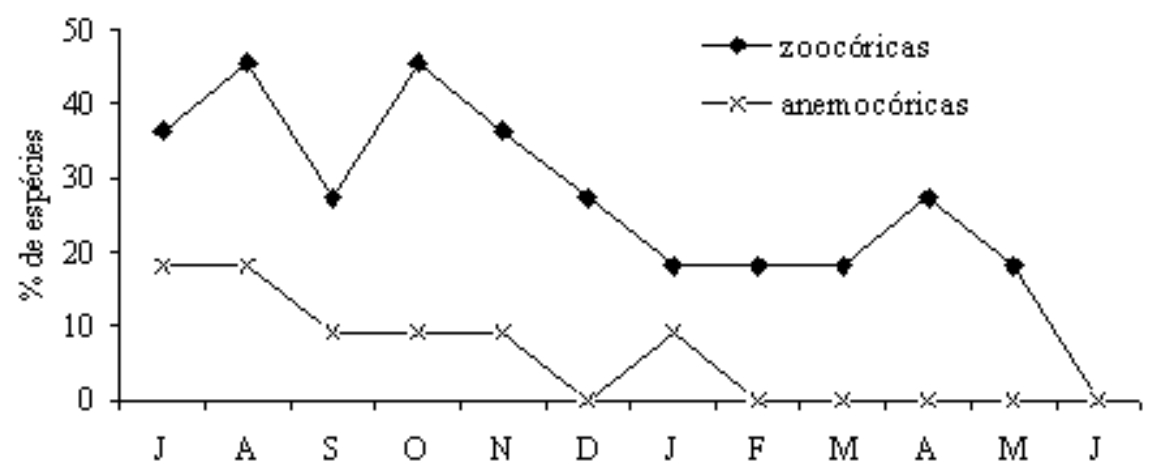

Figura 3 - Porcentagem de espécies em frutificação por modo de dispersão em mata ciliar do rio Formoso, Mato Grosso do Sul, Brasil.

$\square$ comunidade $\mathbf{D}$ Atalea phalerata $\square$ Guibourtia hymenifolia

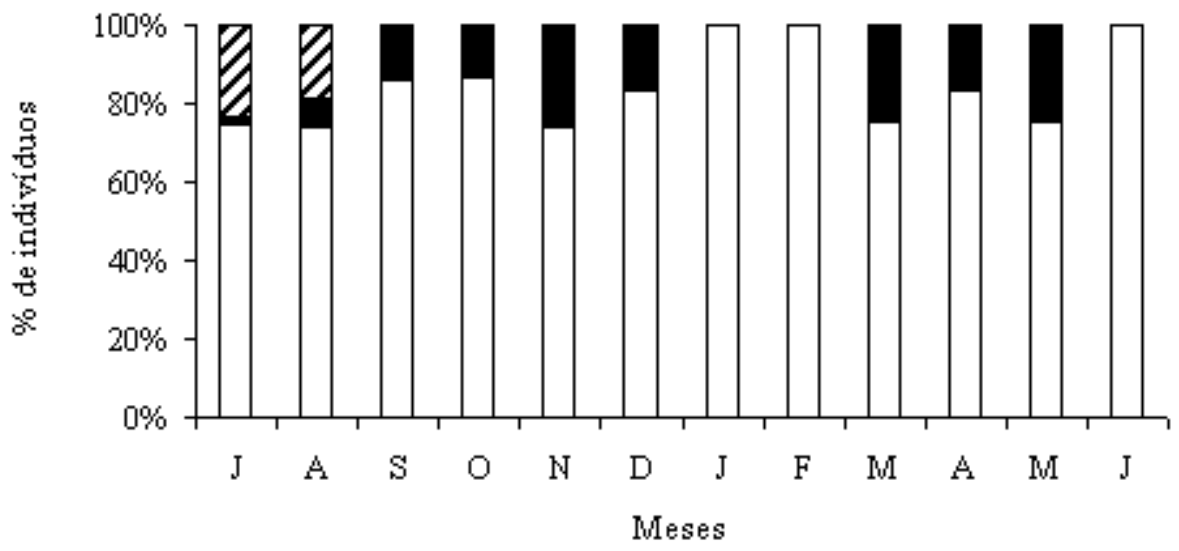

Figura 4 - Porcentagem de indivíduos com frutos maduros da comunidade arbórea e das espécies Guibourtia hymenifolia e Attalea phalerata em mata ciliar do rio Formoso, Mato Grosso do Sul, Brasil.

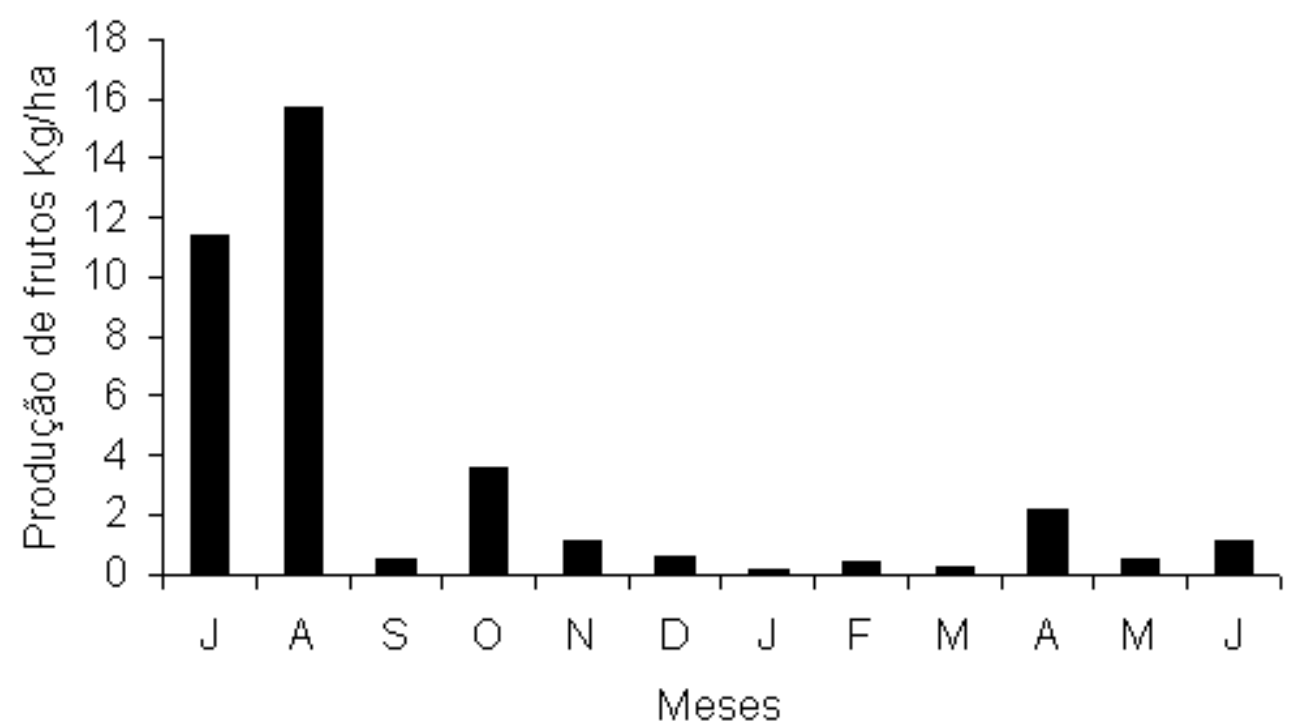

Figura 5 - Produção de frutos da mata ciliar do rio Formoso, Mato Grosso do Sul, Brasil. 
Tabela 1 - Fenologia reprodutiva das espécies da mata ciliar do rio Formoso, estado do Mato Grosso do Sul, Brasil. ANE: Anemocóricas, ZOO: Zoocóricas; A: arbóreo; N: número de indivíduos.

\begin{tabular}{|c|c|c|c|c|c|}
\hline Família / espécie & $\mathrm{N}$ & Hábito & Dispersão & Floração & Frutificação \\
\hline \multicolumn{6}{|l|}{ ANACARDIACEAE } \\
\hline Astronium graveolens Jacq. & 4 & A & ANE & - & - \\
\hline Astronium fraxinifolium Schott & 5 & A & ANE & ago-set & out-nov \\
\hline $\begin{array}{l}\text { Myracrodruon urundeuva Fr. All. } \\
\text { ANNONACEAE }\end{array}$ & 8 & A & AUT & jun-set & set-out \\
\hline $\begin{array}{l}\text { Unonopsis lindimanii Fries. } \\
\text { APOCYNACEAE }\end{array}$ & 46 & A & $\mathrm{ZOO}$ & out & ano todo \\
\hline $\begin{array}{l}\text { Aspidosperma sp. } \\
\text { ARECACEAE }\end{array}$ & 4 & A & ANE & set-out & ago-set \\
\hline $\begin{array}{l}\text { Atallea phalerata Mart. Ex Spreng. } \\
\text { BIGNONIACEAE }\end{array}$ & 121 & A & $\mathrm{ZOO}$ & abr-mai & ago-nov \\
\hline Tabebuia sp. & 4 & A & ANE & jul-set & set-out \\
\hline $\begin{array}{l}\text { Tabebuia impetiginosa (Mart.) Standl. } \\
\text { CECROPIACEAE }\end{array}$ & 3 & A & ANE & ago/00 e mai/01 & set/00 e set/01 \\
\hline $\begin{array}{l}\text { Cecropia pachystachya Trec. } \\
\text { CLUSIACEAE }\end{array}$ & 7 & A & $\mathrm{ZOO}$ & ago-out & nov-fev \\
\hline $\begin{array}{l}\text { Calophyllum brasiliensis Camb. } \\
\text { COMBRETACEAE }\end{array}$ & 3 & A & $\mathrm{ZOO}$ & set-nov & abr-jun \\
\hline Combretum leprosum Mart. & 4 & A & ANE & out-dez & jun-set \\
\hline $\begin{array}{l}\text { Terminalia triflora Griseb. } \\
\text { FABACEAE }\end{array}$ & 3 & A & ANE & jun-jul & ago-set \\
\hline Guibourtia hymenifolia (Moric.) J. Leonard & 9 & A & $\mathrm{ZOO}$ & mai-jun & jul-ago \\
\hline Holocalyx balansae Mich. & 5 & A & $\mathrm{ZOO}$ & out & out-nov \\
\hline Inga cf. marginata Willd. & 3 & A & $\mathrm{ZOO}$ & set & out \\
\hline $\begin{array}{l}\text { Anadenanthera macrocarpa (Benth.) Brenan } \\
\text { LAURACEAE }\end{array}$ & 8 & & ANE & nov/00 & ago-set/01 \\
\hline $\begin{array}{l}\text { Nectandra nitidula Nees. } \\
\text { MELIACEAE }\end{array}$ & 7 & A & $\mathrm{ZOO}$ & set-nov & jan-fev \\
\hline Guarea guidonea (L.) Sleumer & 5 & A & $\mathrm{ZOO}$ & dez-mar & nov-dez \\
\hline Guarea kunthiana A. Juss & 6 & A & $\mathrm{ZOO}$ & ano todo & set \\
\hline Trichilia silvatica D. C. & 2 & A & $\mathrm{ZOO}$ & mar-abr & fev \\
\hline $\begin{array}{l}\text { Trichilia sp. } \\
\text { MORACEAE }\end{array}$ & 3 & A & $\mathrm{ZOO}$ & set-nov & out-dez \\
\hline Ficus pertusa L. F. & 4 & A & $\mathrm{ZOO}$ & mar-abr & $\begin{array}{l}\text { mai-jun/00 e } \\
\text { fev/01 }\end{array}$ \\
\hline Ficus sp. & 3 & A & $\mathrm{ZOO}$ & set & abr e ago \\
\hline Ficus calyptoceras (Miq.) Miq. & 3 & A & $\mathrm{ZOO}$ & nov-dez & mar-abr \\
\hline $\begin{array}{l}\text { Maclura tinctoria (L.) D. Don ex Steud. } \\
\text { MYRTACEAE }\end{array}$ & 1 & A & $\mathrm{ZOO}$ & out & dez \\
\hline Myrcianthes pungens (Berg) Legr. & 65 & A & $\mathrm{ZOO}$ & set & nov-dez \\
\hline $\begin{array}{l}\text { Psidium sartorianum (O. Berg) Nied. } \\
\text { STERCULIACEAE }\end{array}$ & 25 & A & $\mathrm{ZOO}$ & ago & fev-abr \\
\hline $\begin{array}{l}\text { Guazuma ulmifolia Lam. } \\
\text { ULMACEAE }\end{array}$ & 7 & A & $\mathrm{ZOO}$ & set/00 & $\mathrm{abr} / 00$ \\
\hline $\begin{array}{l}\text { Trema micrantha (L.) Blum. } \\
\text { VERBENACEAE }\end{array}$ & 6 & A & $\mathrm{ZOO}$ & set-jan/00 & fev-mar/01 \\
\hline Cytharexyllum mirianthum Cham. & 4 & A & $\mathrm{ZOO}$ & out-dez & nov \\
\hline
\end{tabular}


Tabela 2 - Correlações de Spearman (rs) entre as fenofases estudadas e os fatores climáticos (pluviosidade e temperatura), na mata ciliar do rio Formoso em Bonito Mato Grosso do Sul.(p $£$ 0.05)

\begin{tabular}{ccc}
\hline Fenofases & Temperatura & Pluviosidade \\
\hline Botão & $r s=0,1732 ; p=0,59$ & $r s=0,4034 ; p=0,193$ \\
Antese & $r s=-0,2035 ; p=0,526$ & $r s=-0,1081 ; p=0,738$ \\
Frutos imaturos & $r s=-0,3256 ; p=0,302$ & $r s=-0,1103 ; p=0,733$ \\
Frutos maduros & $r s=-0,3335 ; p=0,289$ & $r s=-0,1428 ; p=0,658$ \\
\hline
\end{tabular}

\subsection{Fenologia}

Foram amostrados 378 indivíduos do estrato arbóreo da mata ciliar com DAP (Diâmetro à Altura do Peito) $\geq$ e” 10 $\mathrm{cm}$, localizados numa faixa de quatro metros a partir do leito do rio, numa trilha de $1.190 \mathrm{~m}$ de comprimento que margeia o rio Formoso em toda sua extensão, dentro dos limites da fazenda. As observações fenológicas foram realizadas de julho de 2000 a julho de 2001, na última semana de cada mês, com o auxílio de um binóculo. A ocorrência das fenofases reprodutivas foi avaliada a partir da observação direta da copa das árvores sendo registrada somente a presença ou ausência de flores e frutos (Bencke \& Morellato 2002). Neste estudo o período de floração foi dividido em botão e antese ou floração propriamente dita (flores abertas) e o período de frutificação em frutos imaturos e frutos maduros ou frutificação (frutos prontos para dispersão, Morellato et al. 1989). As exsicatas das espécies encontram-se depositadas no Herbário Rioclarense (HRCB), da UNESP de Rio Claro.

\subsection{Produção de frutos}

A biomassa de frutos e sementes produzida pela mata ciliar foi estimada quinzenalmente na mesma trilha utilizada para a fenologia, somando uma área de 0,47 ha (trilha de $1190 \mathrm{~m}$ de comprimento e $4 \mathrm{~m}$ de largura), através da coleta, contagem e pesagem de todos os frutos imaturos, maduros e sementes no chão da mata. As espécies foram classificadas quanto aos modos de dispersão de sementes em zoocóricas, anemocóricas e outros, seguindo os critérios propostos por Morellato \& Leitão Filho (1992).

\subsection{Análise dos dados}

A porcentagem de espécies em cada mês nas quatro fenofases reprodutivas foi calculada para descrever os padrões fenológicos da comunidade arbórea e o número de espécies arbóreas por mês em cada fenofase foi correlacionado aos fatores climáticos, temperatura e pluviosidade, do período de estudo através da Correlação de Spearman a fim de verificar se existe relação entre a fenologia e o clima. O Teste de Mann-Whitney foi utilizado para comparar a produção de frutos pela comunidade arbórea nas estações seca e chuvosa.

\section{Resultados}

\subsection{Fenologia reprodutiva}

As 29 espécies amostradas estão distribuídas em 17 famílias, sendo que as mais abundantes em número de indivíduos foram Arecaceae $(n=121)$, Myrtaceae $(n=90)$ e Annonaceae ( $\mathrm{n}=46)$ (Tabela 1).

A comunidade arbórea floresceu e frutificou o ano todo, com maior ocorrência de espécies nas fenofases reprodutivas no final da estação seca e durante a estação úmida, de setembro a novembro, com a frutificação sofrendo uma diminuição de fevereiro a maio (Figura 2). A maior porcentagem de espécies em botão ocorreu em novembro e a de espécies em antese ocorreu na transição entre a estação seca e a chuvosa, em setembro (Figura 2A). A produção de frutos imaturos foi sempre maior do que a de frutos maduros, ambas com pico na estação chuvosa, em outubro (Figura 2B).

Não ocorreu nenhuma correlação significativa entre as fenofases reprodutivas e a pluviosidade e temperatura (Tabela 2). Das 29 espécies estudadas, nove são anemocóricas (31\%) e 20 são zoocóricas (69\%) (Tabela 1). As espécies zoocóricas apresentaram dois picos de frutificação, um no mês de agosto, na estação seca, e outro no mês de outubro, no início da estação chuvosa (Figura 3). A maior parte das espécies anemocóricas frutificou nos meses de julho e agosto (sendo julho o mês mais seco do ano de estudo) (Figura 3). O elevado número de espécies zoocóricas com fruto em julho e pico de frutificação em agosto, pode ser reflexo da grande produção de frutos por Guibourtia hymenifolia (Fabaceae), que amadureceu seus frutos maciçamente neste mês (Figura 4). A espécie de palmeira Attalea phalerata incrementou a produção de frutos da comunidade vegetal em todos os meses do ano de estudo com exceção de janeiro e fevereiro, quando ocorreram apenas frutos imaturos (Figura 4). Esta espécie foi um recurso 
muito importante para a guilda de frugívoros do local de estudo já que produziu frutos durante o ano todo mas, principalmente, na época mais seca do ano quando ocorreu maior escassez de recursos para a fauna (Figura 3).

\subsection{Produção de frutos}

A comunidade vegetal produziu em média $3 \mathrm{~kg} / \mathrm{ha} /$ mês de frutos imaturos e maduros ao longo do ano de estudo (Figura 5). Nos meses de julho e agosto ocorreu uma alta produção de frutos de Guibourtia hymenifolia, com 11,3 $\mathrm{kg} / \mathrm{ha}$ em julho e 15,7 kg/ha em agosto; neste período 99\% dos frutos coletados no chão da mata pertenciam a esta espécie (Figura 4). Este fato fez com que a biomassa de frutos verdes e maduros produzida em julho e agosto ficasse muito maior que aquela dos demais meses. Entretanto, o teste de Mann-Whitney não apontou diferença significativa na produção de frutos pela comunidade arbórea entre as estações seca e chuvosa $(U=14, p=0,16)$.

\section{Discussão}

O ritmo de floração encontrado para a mata ciliar do rio Formoso ficou bem próximo daqueles descritos por Morellato et al.(1989) e Morellato (1991, 1995) para florestas semidecíduas do Estado de São Paulo, por Carmo \& Morellato (2000) para as matas ciliares do rio Tibagi no Paraná, por Oliveira \& Paula (2001) para as matas de galeria do Distrito Federal e por Funch et al. (2002) para a mata de galeria na Chapada Diamantina, no Estado da Bahia. Todas estas comunidades vegetais apresentam período de floração maior na estação úmida (setembro a março) com o pico em outubro, no início das chuvas. Esta semelhança nos padrões de floração pode estar relacionada ao fato de todas as áreas estarem sob clima sazonal, isto é, com estações seca e úmida bem marcadas. A floração na transição estação seca para úmida ou no início da estação úmida, nas florestas semidecíduas, tem sido atribuída, principalmente, à redução do estresse hídrico causado pelas primeiras chuvas após o período de seca, ao aumento do fotoperíodo e à elevação da temperatura (Morellato et al. 1989, Morellato 1991, 1995). Apesar de não se esperar um estresse hídrico acentuado na estação seca para as matas ciliares, estes mesmos fatores podem estar influenciando o padrão de floração observado na mata ciliar do rio Formoso, embora não tenham sido encontradas correlações significativas entre o clima e a fenologia.

O pico de frutificação que ocorreu na estação úmida na mata ciliar do rio Formoso foi semelhante aos padrões de frutificação apresentados por outras matas ciliares (Oliveira \& Paula 2001, Carmo \& Morellato 2000, Funch et al. 2002), mas diferiu do encontrado para florestas semidecíduas (Morellato 1991, 1995, Morellato \& Leitão-Filho 1992, 1996). Esta diferença está relacionada à maior proporção de espécies zoocóricas nas matas ciliares, em torno de 75\%
(Carmo \& Morellato 2001, este estudo) quando comparada às florestas semidecíduas (57\% segundo Morellato 1991, 1995), ficando próximo à proporção encontrada entre espécies de Floresta Atlântica (88\% segundo Morellato et al. 2000) ou da Serra do Japi (70\% segundo Morellato \& Leitão Filho 1992). Durigan (1991) constatou ainda que matas ciliares inseridas em área de cerrado e em florestas semidecíduas possuem 95\% e 75\% de espécies zoocóricas, respectivamente. A partir deste fato é possível verificar a grande importância dessas espécies e o papel fundamental que os animais exercem na dinâmica das matas ciliares.

O padrão de frutificação das espécies zoocóricas encontrado para a mata ciliar do rio Formoso, com frutos maduros disponíveis durante o ano todo e com pico de produção na estação úmida, pode estar associado à manutenção de recursos para os animais dispersores de sementes, como sugerido para outras áreas de floresta tropical (Smythe 1970, Hilty 1980, Wheelwright 1985). Padrão semelhante já foi observado para espécies zoocóricas de floresta semidecídua e de Floresta Atlântica (Morellato et al. 1989, Morellato 1991, 1995, Talora \& Morellato 2000). Já a frutificação das espécies anemocóricas nos meses mais secos do ano (julho e agosto), na mata ciliar estudada, está relacionada às condições ambientais favoráveis na estação seca a dispersão de suas sementes, principalmente a baixa precipitação, baixa umidade relativa e ventos fortes, como sugerido para espécies anemocóricas de florestas semidecíduas por Morellato (1991, 1995), Morellato \& LeitãoFilho (1992, 1996) e Oliveira \& Moreira (1992).

Esta sazonalidade, provavelmente, influencia o comportamento dos frugívoros na mata ciliar do rio Formoso, de forma similar ao descrito por Develey \& Peres (2000) em Floresta Atlântica. Estes autores discutem que um dos fatores que influi na variação de tamanho dos grupos mistos de aves é a distribuição temporal dos recursos alimentares. Embora poucas evidências suportem uma relação entre abundância de frugívoros e sazonalidade, French (1992) relata que mudança no comportamento das aves pode ser uma pressão seletiva importante sobre a produção de frutos. Entretanto, independentemente se são plantas ou animais os responsáveis pela direção das adaptações, as interações que envolvem estes organismos são extremamente importantes para a persistência espacial e temporal das espécies envolvidas (Bond 1994, Feisinger 1987).

Neste sentido, tanto a espécie Guibourtia hymenifolia quanto a palmeira Attalea phalerata apresentaram-se como um recurso muito importante para a fauna associada a mata ciliar do rio Formoso (observação pessoal), pelo fato de terem frutificado maciçamente no período de maior escassez de recursos para os frugívoros. Em especial Guibourtia hymenifolia que apresentou a estratégia de produção maciça de frutos durante o ano de estudo, pode eventualmente se confirmar como espéciechave na manutenção de oferta de frutos para a fauna ao longo do ano. A importância de outras espécies de palmeiras 
e algumas árvores como recurso chave para aves e mamíferos é discutida por Peres (1994, 2000) para floresta de terra firme na Amazônia, por Zona \& Henderson (1989), Terborgh (1986 a, b) em seus trabalhos no Peru e por Galetti \& Aleixo (1998) na Floresta Atlântica.

As matas ciliares foram relativamente pouco estudadas quanto aos aspectos de sua dinâmica e ecologia reprodutiva. Estudos fenológicos que incluam a avaliação da disponibilidade de recursos para a fauna podem contribuir para um melhor entendimento destas formações tão complexas, principalmente devido ao fato das interações animal-planta serem fundamentais para a conservação da biodiversidade. Estudos envolvendo observações sistemáticas em longo prazo para um maior número de espécies são necessários para confirmar os padrões descritos neste e em outros trabalhos, possibilitando compreender a influência dos fatores bióticos e abióticos nos padrões fenológicos das espécies e a dinâmica e conservação deste ecossistema.

\section{Agradecimentos}

Nós agradecemos ao gerente, Laurent Beaudet, e a todos os funcionários da Fazenda Baía Bonita que tornaram possível a realização deste trabalho. Este projeto recebeu recursos das seguintes organizações: Wildlife Conservation Society, Idea Wild, CAPES, CNPq e FAPESP(96/104646 E 01/10300-4). Paula Reys recebeu bolsa de mestrado do CNPq e L. Patrícia C. Morellato e Mauro Galetti são bolsistas de produtividade em pesquisa do CNPq.

\section{Referências bibliográficas}

ASHTON, P.S., T.J. GIVINISH, \& S. APPANAH. 1988. Staggered flowering in the Dipterocarpaceae: new insights into floral induction and the evolution of mast fruiting in the aseasonal tropics. Amer. Naturalist 132:44-66.

BARBOSA, L.M. 2001. Considerações gerais e modelos de recuperação de formações ciliares. In Matas Ciliares: conservação e recuperação (R.R. Rodrigues \& H.F. Leitão Filho, eds.) Edusp, São Paulo, p. 289-311.

BATALHA, M. A. \& MARTINS, M.R. 2004. Reproductive phenology of the cerrado plant community in Emas National Park (central Brazil). Austral. J. Bot. 2: 149-161.

BENCKE, C.C. \& MORELLATO, L.P.C. 2002. Comparação de dois métodos de avaliação da fenologia de plantas, sua interpretação e representação. Revta Brasil. Bot. 25: 269-275.

BERTONI, J.E.A. \& MARTINS, F.R. 1987. Composição florística de uma floresta ripária na Reserva Estadual de Porto Ferreira, SP. Acta Bot. Bras. 1: 17-26.

BOND, W.J. 1994 Do mutualisms matter? Assessing the impact of pollinator and disperser disruption on plant extinction. Philos. Trans. 344: 83-90.
CARMO, M.R.B. \& MORELLATO, L.P.C. 2000. Fenologia de árvores e arbustos das matas ciliares da Bacia do rio Tibagi, Estado do Paraná, Brasil. In Matas Ciliares: conservação e recuperação (R.R. Rodrigues \& H.F. Leitão Filho eds.) Edusp, São Paulo, p. 125-141.

CATHARINO, E.L.M. 1989. Florística de matas ciliares. In Anais do Simpósio sobre Mata Ciliar (L.M. Barbosa coord.). Fundação Cargill, Campinas, p. 61-69.

CHARLES-DOMINIQUE, P., ATRAMENTOWICZ, M., CHARLES-DOMINIQUE, M., GERARD \& PREVOST M.F. 1981. Les mammiferes frugivores arboricoles noctunes dúne forêt guyanaise inter-relations plantesanimaux. Rev. Ecol. (Terre Vie) 35: 341-345.

CROAT, T.B. 1969. Seasonal flowering behavior in Central Panama. Ann. Missouri Bot. Gard. 56: 295-307.

DEVELEY, P.F. \& PERES, C.A. 2000. Resource seasonality and the structure of mixed species bird flocks in a coastal Atlantic forest of southeastern Brazil. J. Trop. Ecol. 16: 33-53.

DURIGAN, G. 1991. Análise comparativa do modo de dispersão das sementes das espécies de cerradão e de mata ciliar no município de Assis/SP. In Simpósio brasileiro de tecnologia de sementes florestais. SMA/ Instituto Florestal, SP p. 278.

FEINSINGER, P. 1987 Effects of plant species on each other's pollination: is community structure influenced? Trends Ecol. Evol 2: 123-126.

FELFILI, J.M. 1997. Dynamics of the natural regeneration in the Gama gallery forest in central Brazil. Forest Ecol. Manag. 91: 235-245.

FELFILI, J.M. \& SILVAJÚNIOR, M.C. 1992. Floristic composition, phytosociology and comparison of cerrado and gallery forests at Fazenda Água Limpa, Federal District, Brazil. In Nature and dynamics of forest-savanna boundaries (Furley P.A., Proctor, J.A. \& Ratter, J.A. eds.) p. 393-415.

FELFILI, J.M., REZENDE, A.V., SILVAJÚNIOR, C. \& SILVA, M.A. 2000. Changes in the floristic composition of cerrado sensu stricto in Brazil over nine-year period. J. Trop. Ecol. 16: 579-590.

FIGLIOLIA, M.B. \& KAGEYAMA, P.Y. 1995. Dispersão de sementes de Inga uruguensis Hook. Et Arn. em floresta ripária do rio Mogi Guaçu, município de Mogi Guaçu SP. Rev. Inst. Florestal 7: 65-80.

FOURNIER, L.A. \& CHARPANTIER, C. 1975. El tamaño de la muestra y la frecuencia de las observaciones en el estudio de las características fenológicas de los arboles tropicales. Turrialba 25: 45-48.

FRENCH, K. 1992. Phenology of fleshy fruits in a wet sclerophyle forest in southe astern Australia: are birds an important influence? Oecologia 90: 366-373.

FUNCH, L. S., FUNCH, R. \& BARROSO, G. M., 2002, Phenology of gallery and montane forest in the Chapada Diamantina, Bahia, Brazil. Biotropica 34: 40-50. 
GALETTI, M. \& ALEIXO, A. 1998. Effects of palm heart harvesting on avian frugivores in the Atlantic rain Forest of Brazil. J. Appl. Ecol. 35: 286-293.

GALETTI, M. \& PEDRONI, F. 1994. Seasonal diet of capuchin monkeys (Cebus apella) in a semideciduous Forest in south-east Brazil. J. Trop. Ecol. 10: 27-39.

GIBBS, P.E. \& LEITÃO-FILHO, H.F. 1978. Floristic composition of an area of gallery Forest near Mogi-Guaçu, state of São Paulo, S.E. Brasil. Revta Brasil. Bot. 1: 151-156.

GRIZ, L.M.S \& MACHADO, I.C. 2001. Fruiting phenology and seed dispersal syndromes in caatinga, a tropical dry forest in the northeast Brazil. J. Trop. Ecol. 17: 303-321.

GUARIMNETO, G., GUARIM, V.L.M.S., MORAES, E.C.C \& FERREIRA, L.A.D. 1996. Fitossociologia de matas ciliares no pantanal mato-grossense. Bol. Mus. Para. Emílio Goeldi 12: 251-263.

HILTY, S.L. 1980. Flowering and fruiting periodicity in a premontane rain forest in pacific Colombia. Biotropica 12: 292-306.

KAGEYAMA, P. \& GANDARA, F.B. 2001. Recuperação de áreas ciliares. In Matas Ciliares: conservação e recuperação (R.R. Rodrigues \& H.F. Leitão Filho, eds.) Edusp, São Paulo, p. 249-269.

KOCHMER, J.P. \& HANDEL, S.N. 1986. Constraints and competition in the evolution of flowering phenology. Ecol. Monogr. 56: 303-325.

KÖEPPEN, W. 1948. Climatologia. Fondo de Cultura Económica, México.

MACEDO, A.C. 1993. Restauração, matas ciliares e de proteção ambiental (J.V. Rezende \& I. Alcântara coords.). Secretaria do Meio Ambiente do Estado de São Paulo, Fundação Florestal, p. 27.

MARIMON, B.S., FELFILI, J.M. \& LIMA, E.S. 2002. Floristics and phytosociology of the gallery Forest of the Bacaba stream, Nova Xavantina, Mato Grosso, Brazil. Edinb. J. Bot. 59: 303-318.

MARIMON, B.S. \& LIMA, E.S. 2001. Caracterização fitofisionômica e levantamento florístico preliminar no Pantanal dos rios Montes-Araguaia, Cocalinho, Mato Grosso, Brasil. Acta Bot. Bras. 15: 213-229.

MARINHO FILHO, J.S. \& REIS, M.L. 1989. A fauna de mamíferos associada às matas ciliares de galeria. In Anais do Simpósio sobre Mata Ciliar (L.M. Barbosa coord.). Campinas SP. Fundação Cargill, p. 43-60.

MORELLATO, L.P.C. 1991. Fenologia de árvores, arbustos e lianas em uma floresta semidecídua no sudeste do Brasil. Tese de doutorado,Universidade de Campinas, Campinas.

MORELLATO, L.P.C. 1995. As estações do ano na floresta. In Ecologia e preservação de uma floresta tropical urbana (P.C. Morellato \& H.F. Leitão-Filho, orgs.). Editora da Unicamp, Campinas p. 37-41.
MORELLATO, L.P.C. \& LEITÃO-FILHO, H.F. 1992. Padrões de frutificação e dispersão na Serra do Japi. In História natural da Serra do Japi: ecologia e presevação de uma área florestal no Sudeste do Brasil (L.P.C. Morellato, org.). Editora da Unicamp/Fapesp, Campinas, p. 112-140.

MORELLATO, L.P.C. \& LEITÃO-FILHO, H.F. 1996. Reproductive phenology of climbers in a Southeastern Brazilian Forest. Biotropica 28: 180-191.

MORELLATO, L.P.C., RODRIGUES, R.R., LEITÃO-FILHO, H.F, JOLY, C.A. 1989. Estudo comparativo de espécies arbóreas de floresta de altitude e floresta mesófila semidecídua na Serra do Japi, Jundiaí, São Paulo. Revta Brasil. Bot. 12: 85-98.

MORELLATO, L.P.C., TALORA, D.C., TAKAHASI A., BENCKE C.C., ROMERA E.C. \& ZIPPARRO V.B. 2000. Phenology of Atlantic Rain Forest trees: A comparative study. Biotropica 32: 811-823.

OLIVEIRA-FILHO, A.T. 1989. Composição florística e estrutura comunitária da floresta de galeria do córrego da Paciência, Cuiabá (MT). Acta Bot. Bras. 3: 91-103.

OLIVEIRA-FILHO, A.T., RATTER, J.A., SHEPHERD, G.J. 1990. Floristic composition and community structure of a central brazilian gallery forest. Flora 184: 103-117.

OLIVEIRA, P.E.A.M. \& MOREIRA, A.G. 1992. Anemocoria em espécies de cerrado e mata de galeria de Brasília, DF. Revta Brasil. Bot. 15: 163-174.

OLIVEIRA, P.E.A.M. \& PAULA F.R. 2001. Fenologia e biologia reprodutiva de plantas de matas de galeria. In Cerrado: caracterização e recuperação de matas de galeria. Brasília, EMBRAPA-CEPAC. P. 303-332.

OPLER, P.A., FRANKIE, GM. \& BAKER H.G. 1976. Rainfall as a factor in the release, timing and synchronization of anthesis by tropical trees and shrubs. J. Biogeogr. 3: 231-236.

PERES, C.A. 1994. composition, density and fruiting phenology of arborescent palms in Amazonian terra firme forest. Biotropica 26: 285-294.

PERES, C.A. 2000. Identifying keystone plant resources in tropical forests: the case of gums from Parkia pods. J. Trop. Ecol. 16:287-317.

PETTS, G.E. 1990. The role of ecotones in aquatic landscape management. In The ecology and management of aquaticterrestrial ecotones (R. Naiman \& Décamps eds.). The Parthenon Publishing Group, Paris, p. 227-261.

RATHCKE, B. \& LACEY, E. P. 1985. Phenological patterns of terrestrial plants. Ann. Rev. Ecol. Syst. 16: 179-214.

RATTER, J.A. 1980. Notes on the vegetation of Fazenda Água Limpa (Brasília - DF, Brazil). Royal Botanic Garden, Edinburgh.

RODRIGUES, R.R. 1989. Análise estrutural das formações ripárias. In Anais do Simpósio sobre Mata Ciliar (L.M. Barbosa coord.). Campinas SP. Fundação Cargill, p. 89-99. 
RODRIGUES, R.R. \& GANDOLFI, S. 2001. Conceitos, tendências e ações para a recuperação de florestas ciliares. In Matas Ciliares: conservação e recuperação (R.R. Rodrigues \& H.F. Leitão Filho, eds.) Edusp, São Paulo, p. 235-247.

SABINO, J. \& CASTRO, R.M.C. 1990. Alimentação, período de atividade e distribuição espacial dos peixes de um riacho da Floresta Atlântica (sudeste do Brasil). Rev. Bras. Biol. 50: 23-36.

SMYTHE, N. 1970. Relationships between fruiting seasons and seed dispersal methods in a neotropical forest. Am. Nat. 104: 25-35.

SNOW, D.W. 1965. A possible selective factor in the evolution of fruiting seasons in tropical forest. Oikos 15: 274-281.

TALORA, D.C. \& MORELLATO, L.P.C. 2000. Fenologia de espécies arbóreas em floresta de planície litorânea do sudeste do Brasil. Revta. Brasil. Bot. 23: 13-26.

TERBORGH, J. 1986a. Keystone plant resources in the tropical forest. In Conservation Biology: the science of scarcity and diversity (M.E. Soulé ed.), Sunderland, Sinauer, p. 330-344.

TERBORGH, J. 1986b. Community aspects of frugivory in tropical forests. In Frugivores and seed dispersal (A. Estrada \& T.H. Fleming, eds). Dr. W. Junk Publishers, Netherlands, p. 371-384.

VAN SCHAIK, C.P. 1986. Phenological changes in a Sumatran rain forest. J. Trop. Ecol. 2: 327-347.

WHEELWRIGHT, N.T. 1985. Competition for dispersers, and the time of flowering and fruiting in a guild of tropical trees. Oikos 44: 465-477.

ZONA, S. \& HENDERSON, A. 1989. Areview of animal mediated seed dispersal of palms. Selbyana 11: 6-21.

Título: Fenologia reprodutiva e disponibilidade de frutos de espécies arbóreas em mata ciliar no rio Formoso, Mato Grosso do Sul

Autores: Paula Reys, Mauro Galetti, L. Patrícia C. Morellato, José Sabino

Biota Neotropica, Vol. 5 ( number 2): 2005

http://www.biotaneotropica.org.br/v5n2/pt/ abstract?short-communication+bn01205022005

Recebido em 10/12/04 - Revisada em 14/06/05.

Publicado em 01/07/2005

ISSN 1676-0603

http://www.biotaneotropica.org.br 